\title{
An association between systolic blood pressure and stroke among patients with impaired consciousness in out-of-hospital emergency settings
}

Taro Irisawa ${ }^{1 *}$, Taku Iwami², Tetsuhisa Kitamura ${ }^{3}$, Chika Nishiyama², Tomohiko Sakai ${ }^{4}$, Kayo Tanigawa-Sugihara², Sumito Hayashida ${ }^{5}$, Tatsuya Nishiuchi ${ }^{6}$, Tadahiko Shiozaki ${ }^{1}$, Osamu Tasaki ${ }^{7}$, Takashi Kawamura², Atsushi Hiraide $^{8}$ and Takeshi Shimazu ${ }^{1}$

\begin{abstract}
Background: Stroke is difficult to diagnose when consciousness is disturbed. However few reports have discussed the clinical predictors of stroke in out-of-hospital emergency settings. This study aims to evaluate the association between initial systolic blood pressure (SBP) value measured by emergency medical service (EMS) and diagnosis of stroke among impaired consciousness patients.

Methods: We included all patients aged 18 years or older who were treated and transported by EMS, and had impaired consciousness (Japan Coma Scale $\geqq 1$ ) in Osaka City (2.7 million), Japan from January 1, 1998 through December 31, 2007. Data were prospectively collected by EMS personnel using a study-specific case report form. Multiple logistic regressions assessed the relationship between initial SBP and stroke and its subtypes adjusted for possible confounding factors.
\end{abstract}

Results: During these 10 years, a total of 1,840,784 emergency patients who were treated and transported by EMS were documented during the study period in Osaka City. Out of 128,678 with impaired consciousness, 106,706 who had prehospital SBP measurements in the field were eligible for our analyses. The proportion of patients with severe impaired consciousness significantly increased from $14.5 \%$ in the $<100 \mathrm{mmHg}$ SBP group to $27.6 \%$ in the $>=200 \mathrm{mmHg}$ SBP group ( $P$ for trend $<0.001$ ). The occurrence of stroke significantly increased with increasing SBP (adjusted odd ratio [AOR] 1.34, 95\% confidence interval [CI] 1.33 to 1.35), and the AOR of the SBP $>=200 \mathrm{mmHg}$ group versus the SBP 101-120 mmHg group was 5.26 (95\% Cl 4.93 to 5.60). The AOR of the SBP $>=200 \mathrm{mmHg}$ group versus the SBP 101-120 mmHg group was 9.76 in subarachnoid hemorrhage (SAH), 16.16 in intracranial hemorrhage $(\mathrm{ICH})$, and 1.52 in ischemic stroke (IS), and the AOR of SAH and ICH was greater than that of IS.

Conclusions: Elevated SBP among emergency patients with impaired consciousness in the field was associated with increased diagnosis of stroke.

Keywords: Systolic blood pressure, Prehospital, Impaired consciousness

\footnotetext{
* Correspondence: taroirisawa@yahoo.co.jp

${ }^{1}$ Department of Traumatology and Acute Critical Medicine, Osaka University Graduate School of Medicine, 2-5 Yamada-oka, Suita, Osaka 565-0871, Japan Full list of author information is available at the end of the article
} 


\section{Background}

Stroke is an important public health problem in the industrialized world [1] and there are 300,000 estimated strokes encounter in the prehospital settings annually Japan [2]. To improve neurologic outcomes after stroke, earlier identification and treatment is most important, but it takes longer time for EMS personnel to transport emergency stroke patients to the stroke centers if EMS personnel could not appropriately recognize these patients [3]. If EMS personnel can discriminate patients with stroke in prehospital settings, these patients can be transported fast to appropriate hospitals that offer advanced treatments such as thrombolytic therapy and interventional radiology.

Importantly, it is difficult to assess neurological findings such as paralysis of stroke in patients with impaired consciousness, and an alternative way to select these patients would, therefore, be needed. Although a lot of studies have showed the positive association between systolic blood pressure (SBP) and the risk of stroke occurrence [4], little is known about the relationship between SBP measured by EMS personnel and the risk of stroke occurrence among patients with impaired consciousness.

Osaka City is a largest urban community in western Japan with approximately 2.7 million population, and approximately 200,000 ambulance runs documented annually since January 1998. The purpose of this study was to evaluate the relationship between SBP measured by EMS in prehospital settings and stroke occurrence among emergency patients with impaired consciousness.

\section{Methods}

\section{Study design, population, and setting}

This is a retrospective, population-based observational study based on the ambulance records of Osaka Municipal Fire Department. The study period was from January 1, 1998 to December 31, 2007. This study was approved by the Ethics Committee of Kyoto University Graduate School of Medicine.

All adult patients aged $>=18$ years who suffered impaired consciousness, and were transported to medical institutions by EMS in Osaka City were enrolled in this study. Diagnoses of stroke and its subtypes such as subarachnoid hemorrhage (SAH), intracranial hemorrhage $(\mathrm{ICH})$, and ischemic stroke (IS) were clinically determined by the physicians caring for the patients in collaboration with the EMS personnel.

\section{Japan Coma Scale}

Table 1 shows Japan Coma Scale (JCS) for grading impaired consciousness [5]. The level of consciousness among emergency patients was recorded by EMS personnel according to JCS. The JCS is a simple way for evaluating neurological disturbance focused on patient's awareness. EMS personnel
Table 1 Japan Coma Scale for grading impaired consciousness

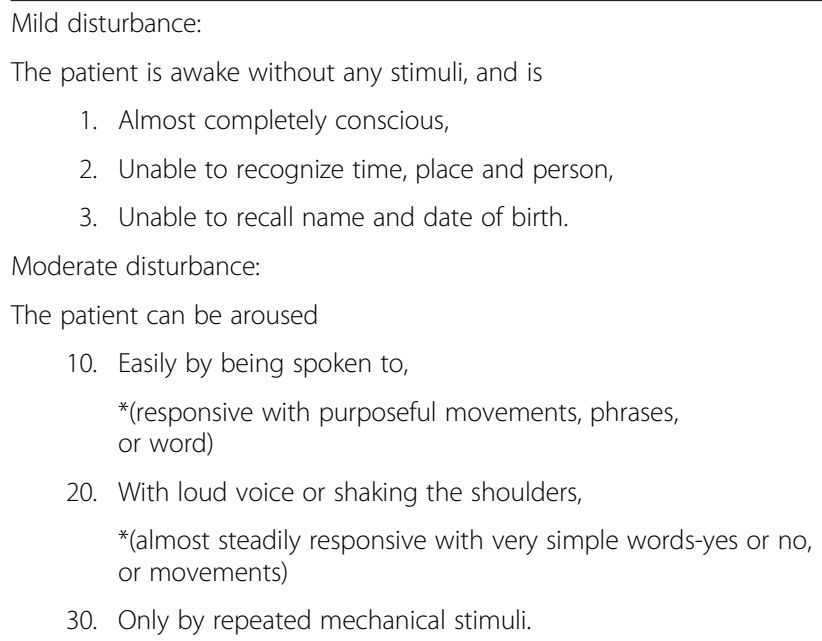

30. Only by repeated mechanical stimuli.

Then, the patient falls into the previous state by cessation of stimulation. Severe disturbance:

The patient cannot be aroused by any forceful mechanical noise stimuli, and

100. Responds with movements to avoid the stimulus,

200. Responds with slight movements including decerebrate and decorticate postures,

300. Does not respond at all except for change in respiratory rhythm.

*Used in patients who cannot open their eyes for any reason. This table was revised from Reference [5].

have generally been using it in prehospital settings. The JCS was roughly divided into the three categories (e.g., mild disturbance, moderate disturbance, and severe disturbance).

\section{Emergency medical service systems and hospitals in Osaka City}

Osaka City, which is a largest urban community in western Japan, has an area of $222 \mathrm{~km}^{2}$, and its population was approximately 2.7 million in 2000 (population density, approximately 12,000 persons $/ \mathrm{km}^{2}$ ). The municipal EMS system has been previously described [6]. Briefly, the EMS system is operated by the Osaka Municipal Fire Department and activated by dialing 119 on the telephone. There were 25 fire stations and a dispatch center in 2007 in Osaka City [7]. Life support is provided 24 hours every day. Usually, each ambulance has a crew of three emergency providers including at least one Emergency LifeSaving Technician (ELST), a highly-trained prehospital emergency care provider. Osaka City included 194 hospitals (34,209 beds) in 2007. Of them, 90 hospitals including 5 critical care centers can accept patients transported by ambulance [8]. 


\section{Data collection and quality control}

Data were uniformly collected using the specific forms that included sex, age, location, vital signs such as first documented systolic and diastolic blood pressure measured manually with sphygmomanometer, heart rate, respiratory rate, and oxygen saturation. The diagnosis was determined by the physician responsible for the care of the patient before admission in the emergency department. The data form was filled out by the EMS personnel in cooperation with the physicians caring for the patient, transferred to the EMS Information Center of Osaka Municipal Fire Department, and then checked by the investigators. If the data sheet was incomplete, the investigators returned it to the relevant EMS personnel for data completion.

\section{Statistical analysis}

The association between the occurrence risk of stroke and SBP (every $20 \mathrm{mmHg}$ ) was "a priori" analyzed considering its subtype (SAH, ICH, or IS). Patient characteristics with and without SBP measurements were evaluated with the use of the $t$-test for numeric variables and the chi-square test for categorical variables. Trends in categorical values and numerical values were tested with logistic regression models and linear tests for trend, respectively. Multiple logistic regression analysis was used to assess the occurrence risk of stroke and its subtype among emergency patients with impaired consciousness by $20 \mathrm{mmHg}$ stratum; Adjusted Odds ratios (AORs) and their 95\% confidence intervals (CIs) were calculated. Potential confounding factors were sex, age, and level of consciousness. In addition, the relationship between prehospital SBP and stroke occurrence by impaired consciousness level was evaluated. Statistical analyses were performed with SPSS statistical package version 17.0 J (SPSS, INC., Chicago, IL). $P$ value of $<0.05$ was considered statistically significant.

\section{Results}

During these 10 years, a total of 1,840,784 emergency patients were documented during the study period in Osaka City (Figure 1). Among 1,463,890 adult patients, 643,141 had medical causes excluding obstetrical and trauma causes, 128,678 yielded an impaired consciousness, and 106,706 with prehospital SBP data were eligible for our analyses.

Table 2 shows the characteristics between 106,706 patients with SBP value and 21,972 patients without SBP value. In patients with SBP, mean men age was 63.1 years, and $54.2 \%$ were male. The proportion of mild impaired consciousness was $70.7 \%$, moderate impaired consciousness $15.9 \%$, and severe impaired consciousness $13.4 \%$, respectively. Forty-nine percent of patients with impaired consciousness were assessed in a private residence. Mean initial SBP was $139.5 \mathrm{mmHg}$. Time interval from call to hospital arrival was 25.1 minutes. Although there were statistically significant differences because of the very large number, the characteristics between the groups were almost similar.

Table 3 shows the characteristics of eligible patients with impaired consciousness by prehospital SBP. As a whole, the proportion of patients with severe impaired consciousness significantly increased from $14.5 \%$ in the $<100 \mathrm{mmHg}$ SBP group to $27.6 \%$ in the $>=200 \mathrm{mmHg}$ SBP group $(P$ for trend $<0.001$ ). Because there was an increase on the proportion of severe disturbance from $10.6 \%$ in the $101-$ $120 \mathrm{mmHg}$ group to $14.5 \%$ in the $=<100 \mathrm{mmHg}$ suggesting that low BP might be a factor in the altered mentation, the group with 101-120 $\mathrm{mmHg}$ SBP was defined as a

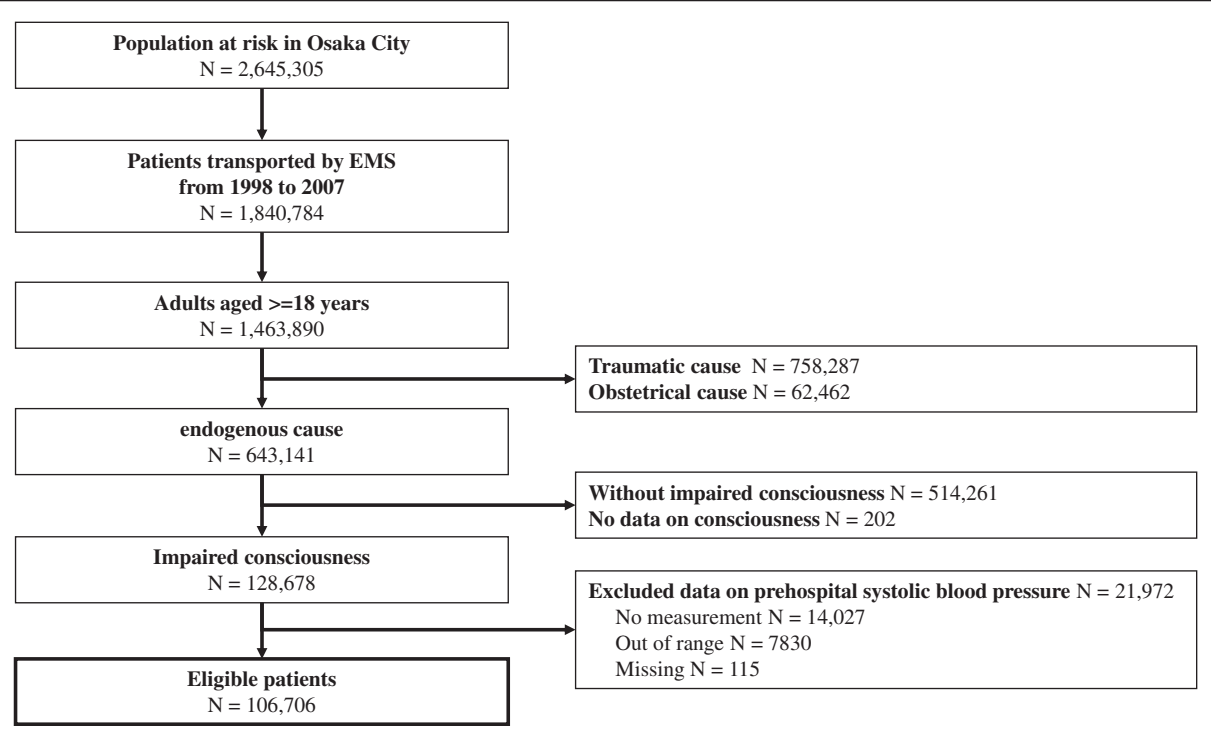

Figure 1 Study flow of emergency patients from January 1, 1998 to December 31, 2007. EMS: emergency medical service. 
Table 2 Characteristics of eligible and non-eligible patients

\begin{tabular}{|c|c|c|c|}
\hline & $\begin{array}{c}\text { Eligible } \\
(\mathrm{N}=106,706)\end{array}$ & $\begin{array}{l}\text { No eligible } \\
(\mathrm{N}=21,972)\end{array}$ & $\begin{array}{c}P \\
\text { value }\end{array}$ \\
\hline Men, n (\%) & $57,879(54.2)$ & $13,341(60.7)$ & $<0.001$ \\
\hline Age, year, mean (SD) & $63.1(20.7)$ & $62.3(20.4)$ & $<0.001$ \\
\hline$>=75$ years, $\mathrm{n}(\%)$ & $37,793(35.4)$ & $7167(32.6)$ & $<0.001$ \\
\hline Consciousness, n (\%) & & & $<0.001$ \\
\hline Mild disturbance & $75,437(70.7)$ & $12,573(57.2)$ & \\
\hline Moderate disturbance & $16,979(15.9)$ & $2121(9.7)$ & \\
\hline Severe disturbance & $14,290(13.4)$ & $7278(33.1)$ & \\
\hline Home, n (\%) & $52,936(49.6)$ & $10,164(46.3)$ & $<0.001$ \\
\hline SBP, mmHg, mean (SD) & $139.5(36.1)$ & - & \\
\hline $\mathrm{DBP}, \mathrm{mmHg}$, mean (SD) & $77.8(21.1)$ & - & \\
\hline HR, counts per a minute, mean (SD) & $89.0(22.3)$ & $70.7(39.9)$ & $<0.001$ \\
\hline $\mathrm{SpO}_{2}, \%$, median (IQR) & 96 (94-98) & $95(88-98)$ & $<0.001$ \\
\hline Call to arrival at the scene, minute, mean (SD) & $6.3(2.5)$ & $6.1(2.5)$ & $<0.001$ \\
\hline Call to contact with patients, minute, mean (SD) & $7.5(2.7)$ & $7.2(2.8)$ & $<0.001$ \\
\hline Call to hospital arrival, minute, mean (SD) & $25.1(8.6)$ & $22.3(8.7)$ & $<0.001$ \\
\hline
\end{tabular}

SBP denotes systolic blood pressure, DBP diastolic blood pressure, HR heart rate; SD standard deviation, IQR inter-quartile range.

reference group to show the relationship between prehospital SBP and stroke occurrence among patients with impaired consciousness.

The proportions of patients with or without stroke according to the SBP were noted in Table 4. Among patients with impaired consciousness, $31.0 \%$ had the proportion of stroke (SAH 1.5\%, ICH 6.3\%, and IS 23.2\%, respectively). This significantly increased from $17.1 \%$ to $63.7 \%$ ( $P$ for trend $<0.001)$. The trends by the subtype of stroke were qualitatively similar.

Figure 2 shows the relationship between SBP measured by EMS in prehospital settings and stroke occurrence among patients with impaired consciousness. The occurrence of stroke significantly increased with increasing SBP (AOR 1.34, 95\% CI 1.33 to 1.35), and the AOR of the $\mathrm{SBP}>=200 \mathrm{mmHg}$ group versus the SBP 101-120 $\mathrm{mmHg}$ group was 5.26 (95\% CI 4.93 to 5.60). In the subgroup analyses in the Figure 3, the AOR for $20 \mathrm{mmHg}$-increment of SBP was 1.48 (95\% CI 1.43 to 1.52$)$ in SAH, 1.69 (95\% CI 1.66 to 1.72 ) in $\mathrm{ICH}$, and 1.14 (95\% CI 1.13 to 1.15 ) in IS, and the AOR of SAH and ICH was greater than that of IS. The AOR of the SBP > $=200 \mathrm{mmHg}$ group versus the SBP $101-120 \mathrm{mmHg}$ group was 9.76 (95\% CI 7.86 to 12.12$)$ in $\mathrm{SAH}, 16.16$ (95\% CI 14.43 to 18.10$)$ in $\mathrm{ICH}$, and 1.52 (1.42 to 1.62 ) in IS, and the AOR of SAH and $\mathrm{ICH}$ was greater than that of IS.

Table 5 shows the relationship between prehospital SBP and stroke occurrence by impaired consciousness level. The AOR of the SBP $>=200 \mathrm{mmHg}$ group versus the SBP 101-120 mmHg group was 16.84 (95\% CI 11.71 to 24.21 ) in mild disturbance and 11.55 (95\% CI 6.70 to 19.90) in moderate disturbance among SAH patients,

Table 3 Characteristics of patients with impaired consciousness according to prehospital systolic blood pressure

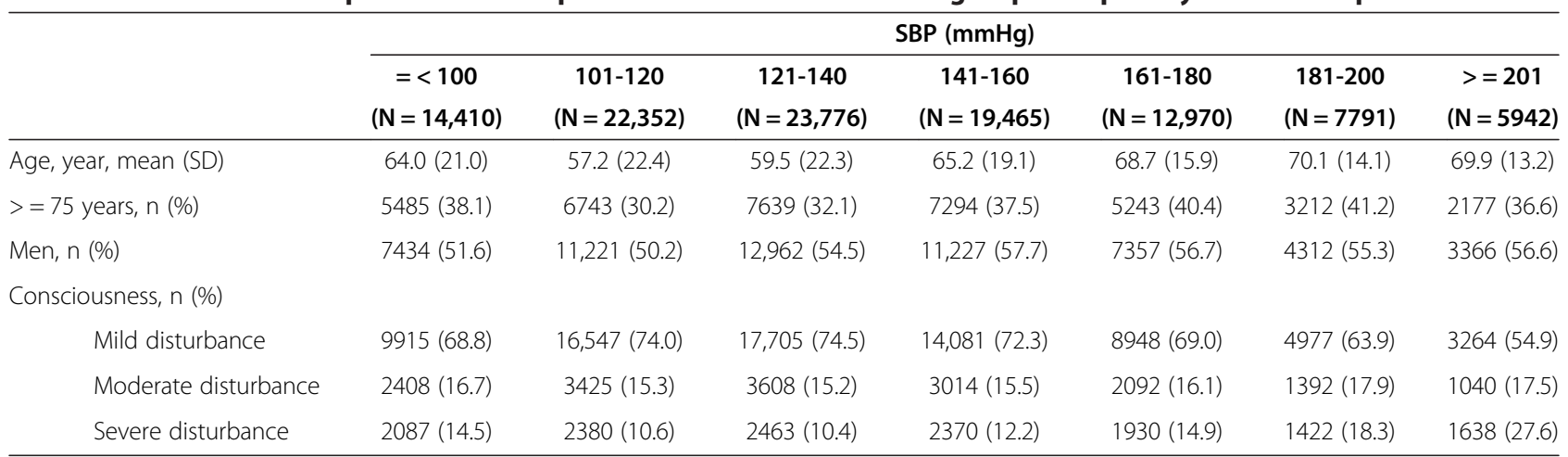

SBP denotes systolic blood pressure, SD standard deviation. 
Table 4 Proportion of stroke patients with impaired consciousness according to prehospital systolic blood pressure

\begin{tabular}{ccccccccc}
\hline & & \multicolumn{7}{c}{ SBP $(\mathbf{m m H g})$} \\
\cline { 3 - 8 } & All & $\mathbf{=}<\mathbf{1 0 0}$ & $\mathbf{1 0 1 - 1 2 0}$ & $\mathbf{1 2 1 - 1 4 0}$ & $\mathbf{1 4 1 - 1 6 0}$ & $\mathbf{1 6 1 - 1 8 0}$ & $\mathbf{1 8 1 - 2 0 0}$ & $>=\mathbf{2 0 1}$ \\
& $(\mathbf{N}=\mathbf{1 0 6 , 7 0 6 )}$ & $\mathbf{( N = 1 4 , 4 1 0 )}$ & $\mathbf{( N = 2 2 , 3 5 2 )}$ & $\mathbf{( N = 2 3 , 7 7 6 )}$ & $\mathbf{( N = 1 9 , 4 6 5 )}$ & $\mathbf{( N = 1 2 , 9 7 0 )}$ & $\mathbf{( N = 7 7 9 1 )}$ & $\mathbf{( N = 5 9 4 2 )}$ \\
\hline Stroke, n (\%) & $33,084(31.0)$ & $2467(17.1)$ & $4515(20.2)$ & $6050(25.4)$ & $6752(34.7)$ & $5506(42.5)$ & $4008(51.4)$ & $3786(63.7)$ \\
SAH, n (\%) & $1631(1.5)$ & $81(0.6)$ & $124(0.6)$ & $243(1.0)$ & $285(1.5)$ & $270(2.1)$ & $286(3.7)$ & $342(5.8)$ \\
ICH, n (\%) & $6699(6.3)$ & $228(1.6)$ & $434(1.9)$ & $709(3.0)$ & $1100(5.7)$ & $1260(9.7)$ & $1274(16.4)$ & $1694(28.5)$ \\
IS, n (\%) & $24,754(23.2)$ & $2158(15.0)$ & $3957(17.7)$ & $5098(21.4)$ & $5367(27.6)$ & $3976(30.7)$ & $2448(31.4)$ & $1750(29.5)$ \\
No stroke, n (\%) & $73,622(69.0)$ & $11,943(82.9)$ & $17,837(79.8)$ & $17,726(74.6)$ & $12,713(65.3)$ & $7464(57.5)$ & $3783(48.6)$ & $2156(36.3)$ \\
\hline
\end{tabular}

SBP denotes systolic blood pressure, SAH subarachnoid hemorrhage, ICH intracranial hemorrhage, IS ischemic stroke.

and 21.19 (95\% CI 17.86 to 25.13 ) in mild disturbance, 13.58 (95\% CI 10.71 to 17.22 ) in moderate disturbance, and 12.61 (95\% CI 10.35 to 15.35$)$ in severe disturbance among ICH patients.

\section{Discussion}

From this large registry of ambulance records, we demonstrated a significant positive relationship between prehospital SBP and the risk of stroke occurrence among emergency patients with impaired consciousness. Although little attention has been paid to SBP measured by EMS in prehospital settings in terms of diagnostic information for stroke, this large population-based registry enabled us to evaluate the relationship between prehospital SBP and stroke occurrence among these patients, and would add new insights on the importance of prehospital SBP measurement. Our results also suggest that prehospital SBP measurements in the patient with impaired conscious level might be a helpful guide as to where to transport a patient especially in communities that have both comprehensive stroke centers and primary ischemic stroke centers.

This study showed that the risk of stroke occurrence among emergency patients with impaired consciousness increased with increasing prehospital SBP. A previous study showed that initial SBP at emergency department arrival was of help for diagnosing intracranial lesion of patients with impaired consciousness [9]. However, diagnosis after hospital arrival is too late to transport the stroke patient to appropriate institution and start treatments against stroke in the effective time window [10]. Guidelines for the Early Management of Adults With Ischemic Stroke by American Heart Association recommend quicker transportation of suspected stroke patients to stroke care units to improve better neurological outcome [11]. Importantly, paralysis of stroke patients is frequently difficult to evaluate when their consciousness is disturbed. Therefore, this study showing the association between prehospital SBP measurements and stroke occurrence among patients with impaired consciousness would contribute to earlier detection of stroke and subsequent rapid transport to appropriate hospitals that can conduct specific treatments for them.

In analyses by stroke subtype, increased SBP was more strongly associated with occurrence of stroke among patients with hemorrhagic brain lesions such as SAH and $\mathrm{ICH}$. The mechanism of hypertensive response among stroke patients is unclear [12] although patients with

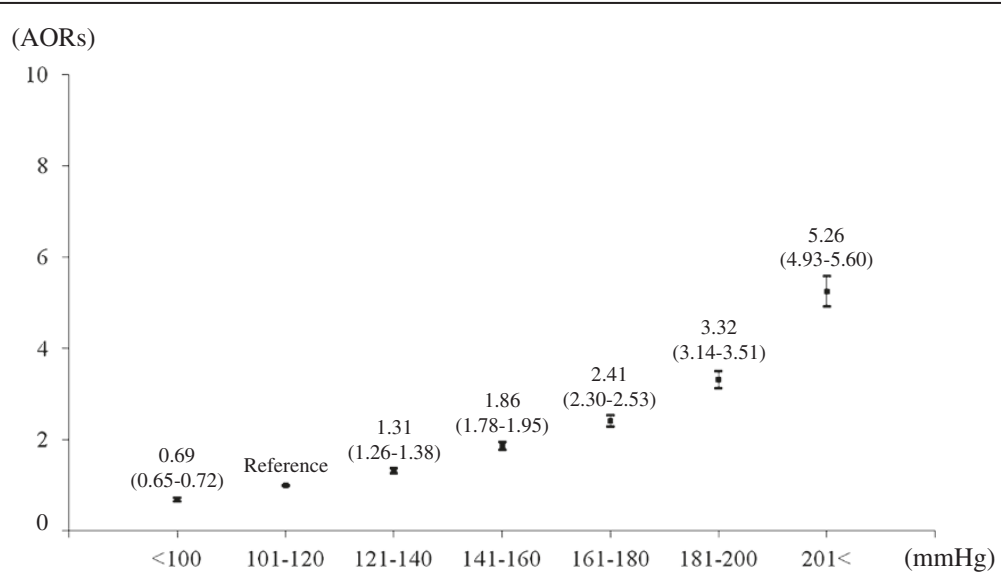

Figure 2 Relationship between SBP measured by EMS personnel in prehospital settings and the risk of stroke occurrence among patients with impaired consciousness. AORs: adjusted odds ratios. 


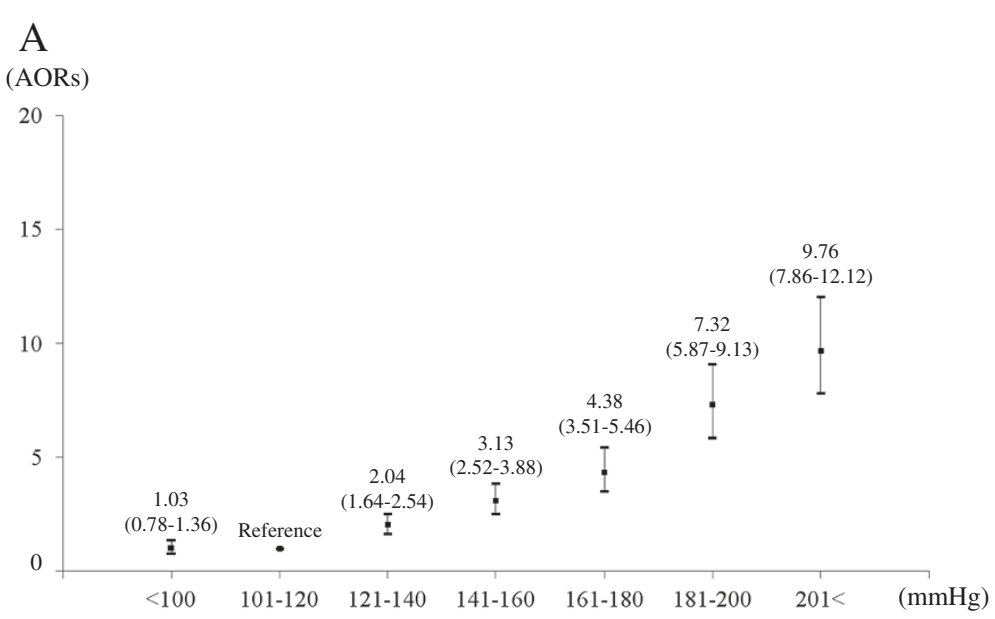

\section{$\mathrm{B}$}

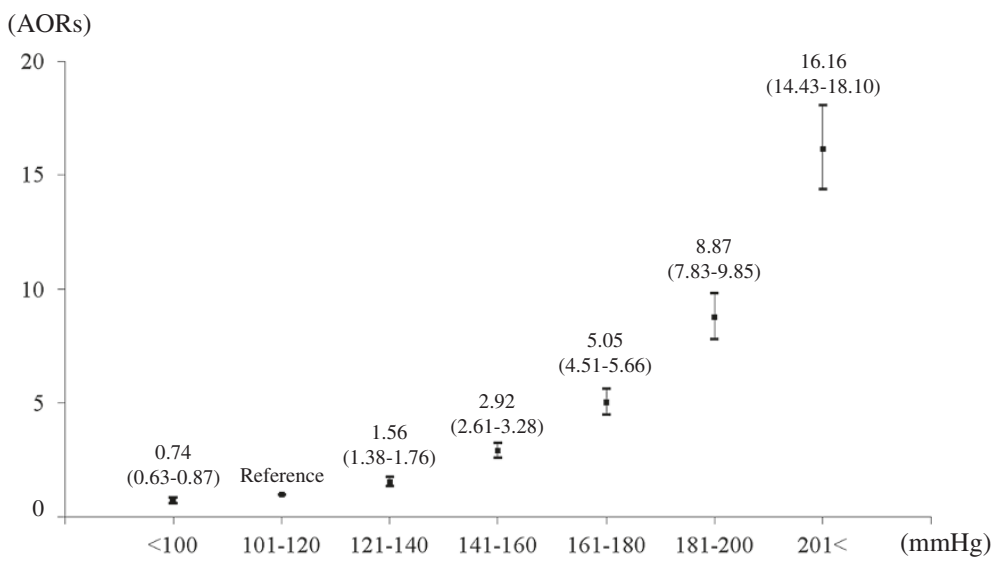

$\mathrm{C}$

(AORs)

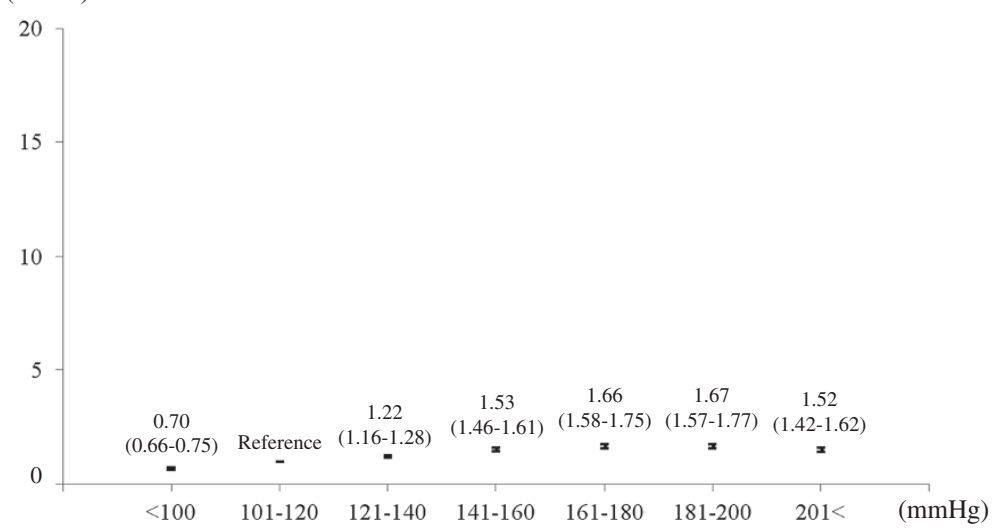

Figure 3 Relationships between SBP measured by EMS personnel in prehospital settings and the risk of stroke occurrence by its stroke subtype among patients with impaired consciousness. (A) SAH, (B) ICH, and (C) IS. AORs; adjusted odds ratios; SAH: subarachnoid

hemorrhage; IS: ischemic stroke; ICH: intracranial hemorrhage.

acute stroke and those with increased intracranial pressure often have hypertension. It was reported that $84 \%$ of patients with stroke had increased blood pressure in the acute phase [13]. The arterial pressure elevation in response to cerebral ischemia is known as the central nervous system ischemic response [14]. In ischemic stroke, hypertension maybe adaptive response to improve perfusion to the ischemic penumbra protecting 
Table 5 Relationship between prehospital SBP and stroke occurrence by impaired consciousness level

\begin{tabular}{|c|c|c|c|c|c|c|c|c|}
\hline & & \multicolumn{7}{|c|}{ SBP $(\mathrm{mmHg})$} \\
\hline & & $=<100$ & $101-120$ & $121-140$ & $141-160$ & $161-180$ & $181-200$ & $>=201$ \\
\hline & e & $0.70(0.65-0.75)$ & Reference & $1.30(1.23-1.38)$ & $1.78(1.68-1.88)$ & $2.31(2.17-2.45)$ & $2.98(2.78-3.20)$ & $4.30(3.96-4.66)$ \\
\hline \multirow[t]{3}{*}{ Stroke } & Moderate disturbance & $0.66(0.58-0.75)$ & Reference & $1.29(1.16-1.44)$ & $2.05(1.84-2.29)$ & $2.55(2.26-2.87)$ & $3.57(3.12-4.08)$ & $6.69(5.72-7.82)$ \\
\hline & Severe disturbance & $70(0.61-0.80)$ & Reference & $1.39(1.23-1.56)$ & $2.03(1.80-2.29)$ & $2.75(2.42-3.12)$ & $4.80(4.15-5.56)$ & $8.21(7.04-9.56)$ \\
\hline & Mild d & $0.99(0.60-1.63)$ & Reference & $2.35(1.66-3.34)$ & $3.75(2.64-5.32)$ & $5.91(4.13-8.45)$ & $8.91(6.14-12.94)$ & $16.84(11.71-24.2$ \\
\hline \multirow[t]{3}{*}{$\mathrm{SAH}$} & Moderate disturbance & $1.01(0.50-2.09)$ & Reference & $2.34(1.36-4.03)$ & $3.59(2.10-6.15)$ & $5.08(2.93-8.78)$ & $9.92(5.81-16.93)$ & .70-19.90) \\
\hline & Severe disturbance & $0.98(0.66-1.46)$ & Reference & $1.72(1.23-2.39)$ & $2.50(1.81-3.45)$ & $3.12(2.25-4.32)$ & $5.35(3.89-7.37)$ & $6.23(4.58-8.48)$ \\
\hline & Mild disturbance & $0.74(0.57-0.96)$ & Reference & $1.76(1.47-2.10)$ & $3.17(2.67-3.76)$ & $5.82(4.91-6.90)$ & $10.90(9.17-12.94)$ & $21.19(17.86-25.1$ \\
\hline \multirow[t]{3}{*}{$\mathrm{ICH}$} & Moderate disturbance & $0.45(0.31-0.67)$ & Reference & $1.14(0.88-1.48)$ & $2.68(2.11-3.39)$ & $4.64(3.67-5.87)$ & $7.19(5.66-9.14)$ & $13.58(10.71-17.2$ \\
\hline & Severe disturbance & $0.88(0.68-1.13)$ & Reference & $1.62(1.31-2.01)$ & $2.80(2.28-3.43)$ & $4.35(3.55-5.33)$ & $7.34(5.98-9.00)$ & $12.61(10.35-15.3$ \\
\hline & Mild disturbance & $0.70(0.65-0.75)$ & Reference & $1.23(1.16-1.30)$ & 1.59 (1.47-1.65) & 1.77 (1.67-1.89) & $1.83(1.70-1.97)$ & $1.75(1.60-1.90)$ \\
\hline \multirow[t]{2}{*}{ IS } & Moderate disturbance & $0.70(0.61-0.81)$ & Reference & $1.26(1.12-1.41)$ & $1.65(1.47-1.86)$ & $1.63(1.44-1.85)$ & $1.68(1.46-1.93)$ & $1.84(1.58-2.15)$ \\
\hline & Severe disturbance & $0.67(0.58-0.78)$ & Reference & $1.15(1.00-1.31)$ & $1.27(1.12-1.45)$ & $1.26(1.10-1.44)$ & $1.16(1.00-1.34)$ & $0.91(0.78-1.06)$ \\
\hline
\end{tabular}

SBP denotes systolic blood pressure, SAH subarachnoid hemorrhage, $\mathrm{ICH}$, intracranial hemorrhage, IS ischemic stroke. Odds ratio were adjusted for sex and age.

the brain from further ischemia. On the other hand, hypertension in hemorrhagic brain lesion like $\mathrm{SAH}$ or $\mathrm{ICH}$ may cause further damage by worsening cerebral edema, increasing intracranial pressure, or causing hematoma expansion $[15,16]$. Our result showing difference by the subtype of stroke might be partially explained by such pathophysiological differences between hemorrhagic and ischemic lesions.

From our results, emergency patients with impaired consciousness and high SBP should be considered to be transported to the comprehensive stroke centers with capabilities of either neurosurgery or tissue plasminogen activator ( $t-\mathrm{PA})$ administration because these patients might have stroke but prehospital EMS personnel could not distinguish brain hemorrhagic lesions from ischemic ones. In addition, this study showed the strong relationship between high prehospital SBP and the occurrence of $\mathrm{SAH}$ and $\mathrm{ICH}$, and those patients should be treated as quick as possible in order to prevent re-rupture of aneurysms and recurrent bleeding $[17,18]$. Especially, the strength of association between SBP and stroke subtype by impaired conscious level was very powerful with $\mathrm{ICH}$ and to some extent with SAH (mild and moderate disturbances), which would suggest that prehospital SBP can be an important triage guide for selecting patients. Further studies identifying an accurate cutoff point in this regard for SBP in conjunction with level of consciousness would make the EMS triage decision more precise and reduces the risk of overwhelming comprehensive stroke centers with patients that do not need the advanced capabilities. On the other hand, the relationship between SBP in prehospital settings and the occurrence of IS was relatively small. Therefore, to improve positive predictive value for IS patients who are most treatable, development of additional clinical indicators should be found out to make it possible to transport patients with IS to the primary ischemic stroke centers where only t-PA administration could be performed.

There were some limitations to this study. First, in Japan, EMS personnel evaluated level of consciousness among prehospital emergency patients by using JCS rather than the commonly-used Glasgow Coma Scale (GCS). JCS is not preferable to GCS as a consciousness evaluation system in the acute phase of SAH [19]. However, traditionally in Japanese prehospital setting, EMS has been evaluating stroke patients with impaired consciousness by JCS. Although our study cannot compare with GCS directly, these results should, nevertheless, provide useful information on the relationship between SBP and stroke occurrence among patients with impaired consciousness. Second, this study did not obtain data on advanced treatments and neurological outcomes among stroke patients after hospital arrival. Third, data on patient's past history and medications that might affect the occurrence of stroke was lacking. Fourth, we did not obtain information on other diagnosis that could mimic stroke such as hypoglycemia, complicated migraine, prolonged seizures, and subdural hematomas. Finally, there might be unmeasured confounding factors and selection bias that might have influenced the relationship between prehospital SBP and stroke occurrence among emergency patients with impaired consciousness.

\section{Conclusions}

Elevated SBP among emergency patients with impaired consciousness in the field was associated with the increased risk of stroke. Additional research is necessary to determine if field diagnosis of stroke with measuring $\mathrm{BP}$ improves procession of care or outcome. 


\section{Abbreviations}

SBP: Systemic blood pressure; EMS: Emergency medical service; AOR: Adjusted odds ratio; Cl: Confidence interval; SAH: Subarachnoid hemorrhage; IS: Ischemic stroke; ICH: Intracranial hemorrhage; JCS: Japan come scale; ELST: Emergency life-saving technician; CIs: Confidence intervals; GCS: Glasgow Come Scale.

\section{Competing interests}

The authors declare that they have no competing interests.

\section{Authors' contributions}

TI, TI, TK, CN, TS, KT, SH, TN, TS, OT, TK, AH, and TS participated in the idea formation, study design, data analyses, interpretation of results and writing of the report. All the authors read and approved the final manuscript.

\section{Acknowledgments}

We are greatly indebted to all of the EMS personnel at the Osaka Municipal Fire Department and concerned physicians in Osaka City for their indispensable cooperation and support.

\section{Financial support}

This research was supported by a grant for Emergency Management Scientific Research from the Fire Disaster Management Agency (Study concerning strategy for applying the results of Utstein report for improvement of emergency service). The study sponsors had no involvement in the study design, in the collection, analysis and interpretation of data, in the writing of the manuscript or in the decision to submit the manuscript for publication.

\section{Author details}

${ }^{1}$ Department of Traumatology and Acute Critical Medicine, Osaka University Graduate School of Medicine, 2-5 Yamada-oka, Suita, Osaka 565-0871, Japan. ${ }^{2}$ Health Services, Kyoto University, Yoshida-Honmachi, Sakyo-ku, Kyoto 606-8501, Japan. ${ }^{3}$ Department of Social and Environmental Medicine, Graduate School of Medicine, Division of Environmental Medicine and Population Sciences, Osaka University, 2-5 Yamada-oka, Suita, Osaka 565-0871, Japan. ${ }^{4}$ Department of Trauma and Critical Care Medicine and Burn Centers, Social Insurance Chukyo Hospital, 1-1-10 Sanjyo Minamiku, Nagoya, Aichi 457-8510, Japan. ${ }^{5}$ Osaka Municipal Fire Department, 1-12-54 Kujo minami, Nishi-ku, Osaka 550-8566, Japan. ${ }^{6}$ Department of Critical Care and Emergency Medicine, Osaka City University Graduate School of Medicine, 1-5-17 Asahimachi, Abeno-ku, Osaka 545-8585, Japan. ${ }^{7}$ Nagasaki University Hospital Emegency Medical Center, 1-7-1, Sakamoto, Nagasaki, Nagasaki 852-8501, Japan. ${ }^{8}$ Department of acute Medicine, Kinki University Faculty of Medicine, 377-2 Ouno higashi Osaka-Sayama, Osaka 589-8511, Japan.

Received: 14 June 2013 Accepted: 12 December 2013 Published: 17 December 2013

\section{References}

1. Feigin VL, Lawes CM, Bennett DA, Barker-Collo SL, Parag V: Worldwide stroke incidence and early case fatality reported in 56 population-based studies: a systematic review. Lancet Neurol 2009, 8:355-369.

2. Fire and Disaster Management Agency of Japan: Report on the number of patients transported by ambulances according to the disease category. http://www.fdma.go.jp/html/hakusho/h23/h23/html/2-2-4-1_4.html (Accessed January 10, 2013) (in Japanese).

3. Ramanujam P, Castillo E, Patel E, Vilke G, Wilson MP, Dunford JV: Pre-hospital transport time intervals for acute stroke patients. J Emerg Med 2009, 37:40-45.

4. Hashimoto T, Kikuya M, Ohkubo T, Satoh M, Metoki H, Inoue R, Asatama K, Kanoo A, Obara T, Hirose T, Hara A, Hoshi H, Totsune K, Satoh H, Imai Y: Home blood pressure level, blood pressure variability, smoking, and stroke risk in Japanese Men: the Ohasama study. Am J Hypertens 2012, 25:883-891.

5. Ono K, Wada K, Takahara T, Shirotani T: Indications for computed tomography in patients with mild head injury. Neurol Med Chir (Tokyo) 2007, 47:291-298.

6. Kajino K, Iwami T, Kitamura T, Daya M, Ong MEH, Nishiuchi T, Hayashi Y, Sakai T, Shimazu T, Hiraide A, Kishi M, Yamayoshi S: Comparison of supraglottic airway versus endotracheal intubation for the pre-hospital treatment of out-of-hospital cardiac arrest. Critical Care 2011, 15:R236.

7. Osaka Municipal Fire Department: The list of fire departments in Osaka City. http://www.city.osaka.lg.jp/shobo/page/0000018935.htm (Accessed January 10, 2013) (in Japanese).

8. Osaka Medical Facilities Information System: The list of emergency hospitals in Osaka. http://www.mfis.pref.osaka.jp/qq27scripts/qq/ fm27qrinsm out.asp (Accessed January 10, 2013) (in Japanese).

9. Ikeda M, Matsunaga T, Irabu N, Yoshida S: Using vital signs to diagnose impaired consciousness: cross sectional observational study. BMJ 2002, 325:800.

10. Qureshi Al, Kirmani JF, Sayed MA, Safdar A, Ahmed S, Ferguson R, Hershey LA, Qazi KJ, the Buffalo Metropolitan Area and Erie County Stroke Study Group: Time to hospital arrival, use of thrombolytics, and in-hospital outcomes in ischemic stroke. Neurology 2005, 64:2115-2120.

11. Adams HP Jr, del Zoppo G, Alberts MJ, Bhatt DL, Brass L, Furlan A, Grubb RL, Higashida RT, Jauch EC, Kidwell C: Guidelines for the early management of adults with ischemic stroke: a guideline from the American Heart Association/American Stroke Association Stroke Council, Clinical Cardiology Council, Cardiovascular Radiology and Intervention Council, and the Atherosclerotic Peripheral Vascular Disease and Quality of Care Outcomes in Research Interdisciplinary Working Groups: the American Academy of Neurology affirms the value of this guideline as an educational tool for neurologists. Stroke 2007, 38:1655-1711.

12. Donovan AL, Flexman AM, Gelb AW: Blood pressure management in stroke. Curr Opin Anesthesiol 2012, 25:516-522.

13. Wallace JD, Levy LL: Blood pressure after stroke. JAMA 1981, 246:2177-2180.

14. Guyton AC, Hall JE: Textbook of medical physiology. 12th edition. Philadelphia: WB Sauders; 2011:209-220.

15. Semplicini A, Maresca A, Boscolo G, Sartori M, Rocchi R, Giantin V, Forte PL, Pessina AC: Hypertension in acute ischemic stroke: a compensatory mechanism or an additional damaging factor? Arch Intern med 2003 163:211-216.

16. Qureshi Al, Ezzeddine MA, Nasar A, Suri MF, Kirmani JF, Hussein HM, Diviani AA, Reddi AS: Prevalence of elevated blood pressure in 563704 adult patients with stroke presenting to the ED in the United States. Am J Emerg Med 2007, 25:32-38.

17. Lewis BM: Guidelines for the management of spontaneous intracerebral hemorrhage. Stroke 2010, 41:2108-2129.

18. Alexander G: Treatment of ruptured aneurysms: earlier is better. World Neurosurgery 2012, 77:263-265.

19. Takagi K, Aoki M, Ishii T, Nagashima Y, Narita K, Nakagomi T, Tamura A, Yasui N, Hadeishi H, Taneda M, Sano K: Japan Coma Scale as a grading scale of subarachnoid hemorrhage: a way to determine the scale. No Shinkei Geka 1998, 26:509-515 (in Japanese).

\section{doi:10.1186/1471-227X-13-24}

Cite this article as: Irisawa et al:: An association between systolic blood pressure and stroke among patients with impaired consciousness in out-of-hospital emergency settings. BMC Emergency Medicine 2013 13:24

\section{Submit your next manuscript to BioMed Central and take full advantage of:}

- Convenient online submission

- Thorough peer review

- No space constraints or color figure charges

- Immediate publication on acceptance

- Inclusion in PubMed, CAS, Scopus and Google Scholar

- Research which is freely available for redistribution 